\title{
Optic neuritis and acute anterior uveitis associated with influenza $A$ infection: a case report
}

\author{
Hayate Nakagawa \\ Hidetaka Noma \\ Osamu Kotake \\ Ryosuke Motohashi \\ Kanako Yasuda \\ Masahiko Shimura
}

Department of Ophthalmology, Tokyo Medical University Hachioji Medical

Center, Tokyo, Japan
Correspondence: Hayate Nakagawa Department of Ophthalmology, Tokyo Medical University Hachioji Medical Center, II63 Tatemachi, Hachiouji-shi, Tokyo 1930944, Japan

$\mathrm{Tel}+8 \mathrm{I} 42665561 \mathrm{I}$

Fax +8I 426655639

Email rocklifeI03।@yahoo.co.jp
This article was published in the following Dove Press journal:

International Medical Case Reports Journal

4 January 2017

Number of times this article has been viewed

Background: A few reports have described ocular complications of influenza A infection, such as impaired ocular movement, parasympathetic ocular nerve, keratitis, macular lesion, and frosted branch angiitis. We encountered a rare case of acute anterior uveitis and optic neuritis associated with influenza A infection.

Case presentation: A 70-year-old man presented with symptoms of upper respiratory tract infection. A rapid diagnostic test showed a positive result for influenza A. At the same time, he developed ocular symptoms including blurred vision with optic disk edema and hemorrhage in the left eye, and bilateral red eyes. Multiplex polymerase chain reaction performed on aqueous humor sample detected no viral infection. Visual field testing with a Goldmann perimeter showed central and paracentral scotomas in the left eye. In addition to antiviral agent (oseltamivir phosphate $75 \mathrm{mg}$ ), the patient was prescribed topical prednisolone acetate ophthalmic suspension eye drops every 5 hours and high-dose intravenous methylprednisolone $1,000 \mathrm{mg}$ daily for 3 days. Two months later, his best-corrected visual acuity improved to 20/50 with regression of visual field defects in his left eye. Conclusion: We report a case of bilateral acute anterior uveitis and unilateral optic neuritis concomitant with influenza A infection. Topical and systemic corticosteroids were effective to resolve acute anterior uveitis and neuritis. Analysis of aqueous humor sample suggested that acute anterior uveitis and optic neuritis in this case were not caused by influenza A virus infection per se but by autoimmune mechanism.

Keywords: optic neuritis, anterior uveitis, influenza virus, multiplex polymerase chain reaction

\section{Introduction}

Influenza viruses are highly contagious and affect individuals of all ages. Most winter epidemics in humans are caused by types A and B. ${ }^{1}$ Common symptoms of influenza A infection are fever, respiratory symptoms, general weakness, myalgia, headache, and gastrointestinal symptoms. Severe complications include respiratory failure, neurological complications, shock, and even death. However, there are few reports on ocular complications of influenza A infection. These reports described symptoms such as impaired ocular movement, parasympathetic ocular nerve, keratitis, macular lesion, and frosted branch angiitis. ${ }^{2-6}$ We encountered a rare case of acute anterior uveitis and optic neuritis associated with influenza $\mathrm{A}$ infection, and analysis of aqueous humor sample during acute infection revealed interesting results.

\section{Case presentation}

Informed consent was obtained from this patient and this study was approved by the institutional review board of Tokyo Medical University Hachioji Medical Center. 
A 70-year-old man presented with symptoms of upper respiratory tract infection including cough, sore throat, and rhinorrhea. A rapid influenza diagnostic test revealed a positive result for type A influenza. An oral antiviral agent, oseltamivir phosphate $75 \mathrm{mg}$ (Tamiflu ${ }^{\circledR}$; Chugai Pharmaceutical Co., Ltd., Chūō, Tokyo, Japan), was administered twice per day for 5 days, with a smooth course of recovery. At onset, he also developed ocular symptoms including blurred vision in the left eye and conjunctivitis in bilateral eyes.

At the initial ocular examination, his best-corrected visual acuity (BCVA) was 20/25 in the right eye and counting fingers in the left eye. The intraocular pressure was $8 \mathrm{mmHg}$ in the right eye and $16 \mathrm{mmHg}$ in the left eye. Slit-lamp biomicroscopy showed more than one cell in the anterior chamber, fine keratic precipitates and conjunctivitis in both eyes (Figure 1A), and bilateral shallow anterior chamber. The presence of a relative afferent pupillary defect in the left eye was identified by performing the swinging flashlight test. Funduscopy revealed mild optic disk edema and disk hemorrhage in the left eye (Figure 1B). Fluorescein angiography showed dye leakage at the optic disk (Figure 1C). Visual field testing with a Goldmann perimeter showed central and paracentral scotomas in the left eye (Figure 2A). On a full-field electroretinogram (ERG), reductions in the amplitude of oscillatory potentials and b-wave were observed (Figure 3A). Optical coherence tomography (OCT) showed no abnormal findings in bilateral foveae (Figure 1D). Magnetic resonance imaging of the head showed normal optic nerves. The other physical and neurological examinations were unremarkable. Immunochemical, hematological, and biochemical examinations were normal. No viruses including influenza virus (influenza virus $\mathrm{A}$ and $\mathrm{B}$, herpes simplex virus-1 and -2, varicella-zoster virus, cytomegalovirus, parvovirus B19, human herpesvirus-6, -7, and -8, BK virus, JC virus, and Epstein-Barr virus) were detected by multiplex polymerase chain reaction performed in aqueous humor sampled from the anterior chamber of both eyes. Under a diagnosis of bilateral acute anterior uveitis and optic

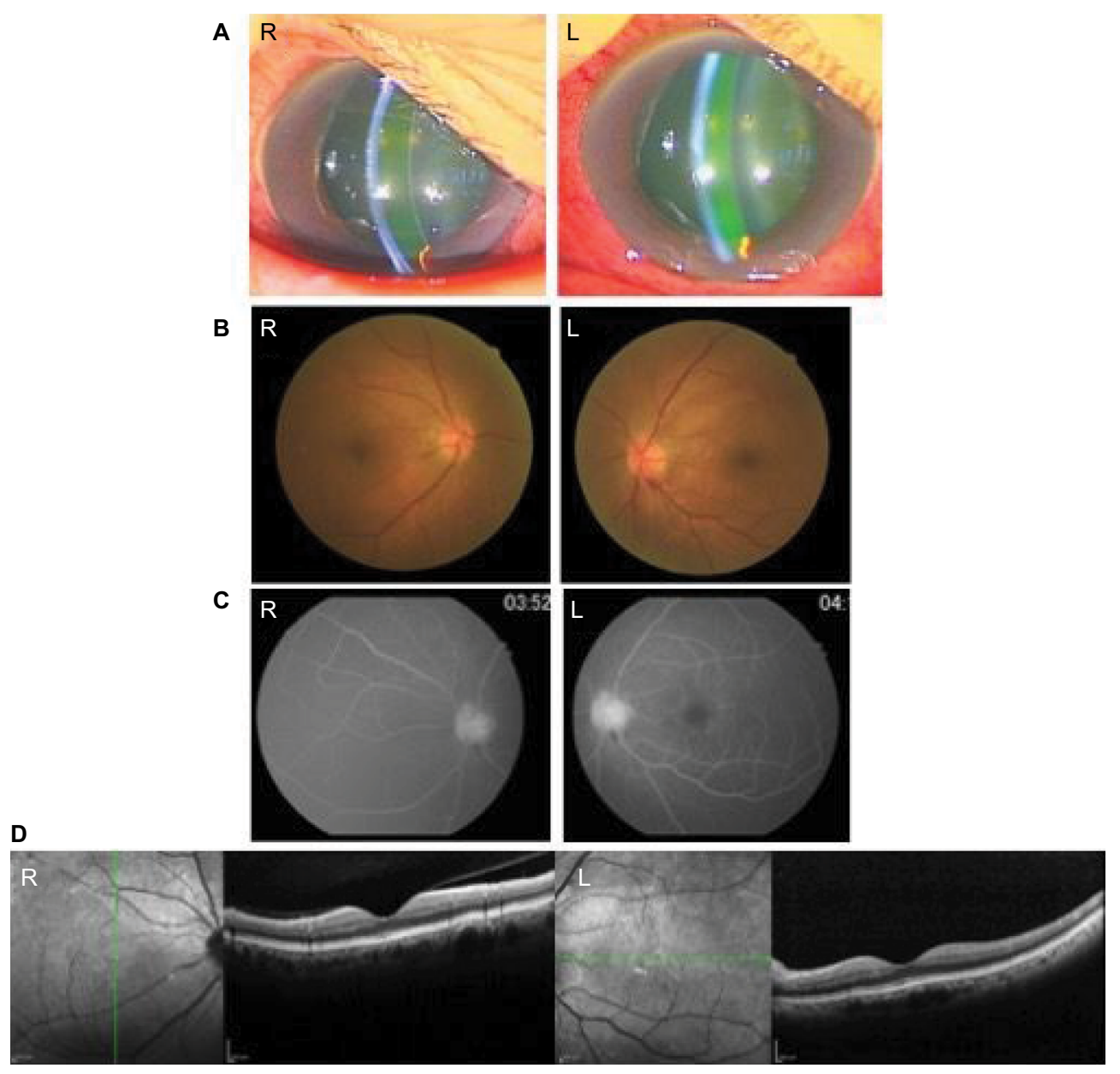

Figure I Initial ocular manifestations.

Notes: (A) Slit-lamp photographs showed anterior chamber inflammation and severe conjunctivitis in both eyes. Fluorescein leakage was observed in the anterior chamber. (B) Fundus photographs showed mild optic disk edema and disk hemorrhage in the left eye. (C) Fluorescein angiographic images showed dye leakage at the optic disk in the left eye. (D) Optical coherence tomographic (OCT) images showed no abnormal foveal findings in both eyes.

Abbreviations: $R$, right; L, left. 


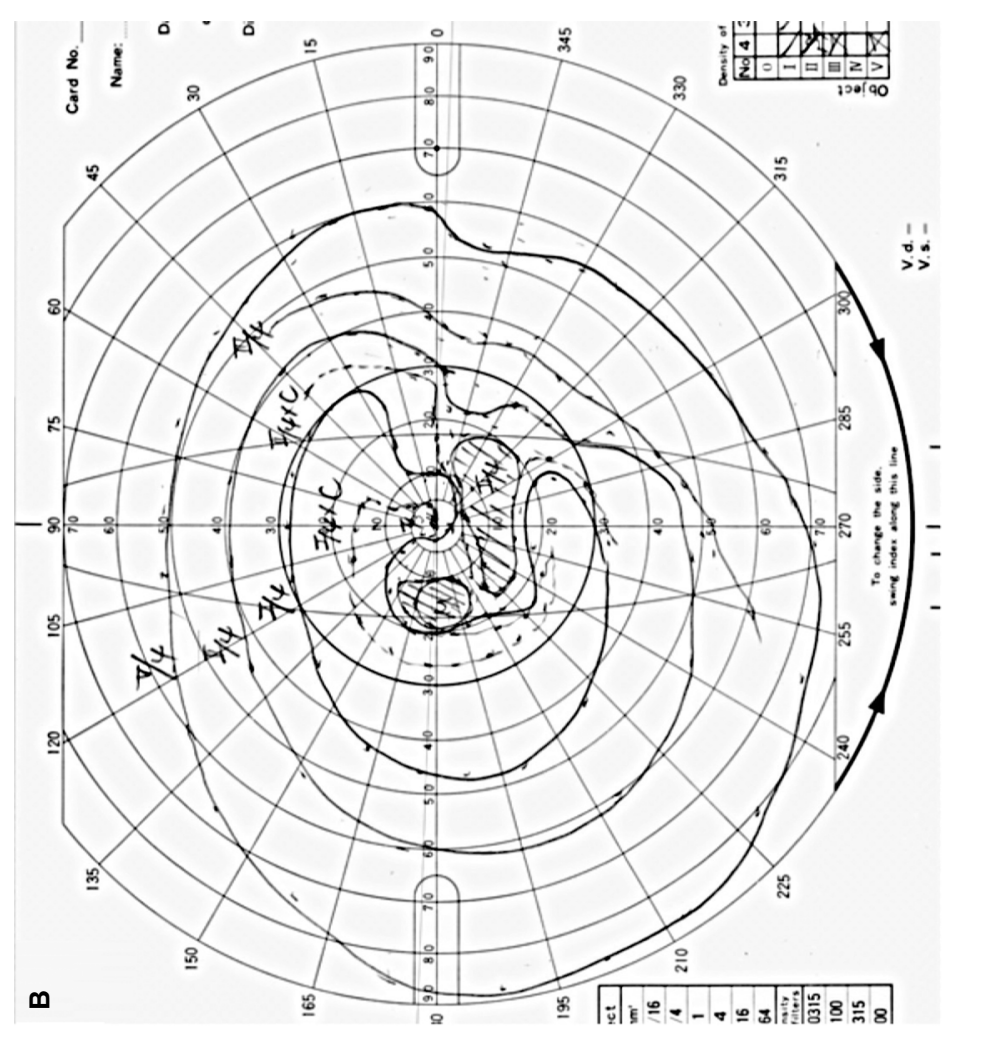

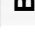

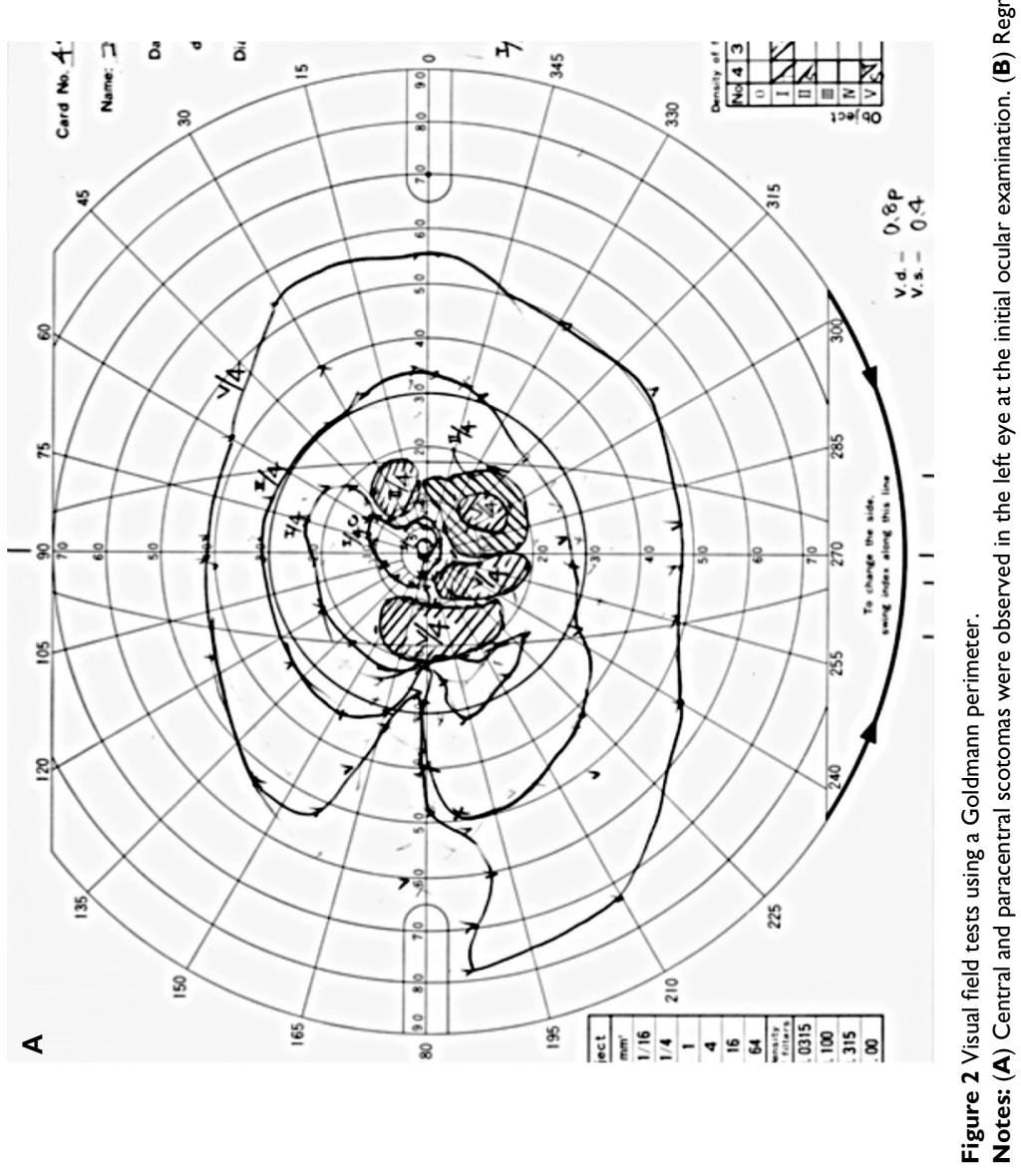




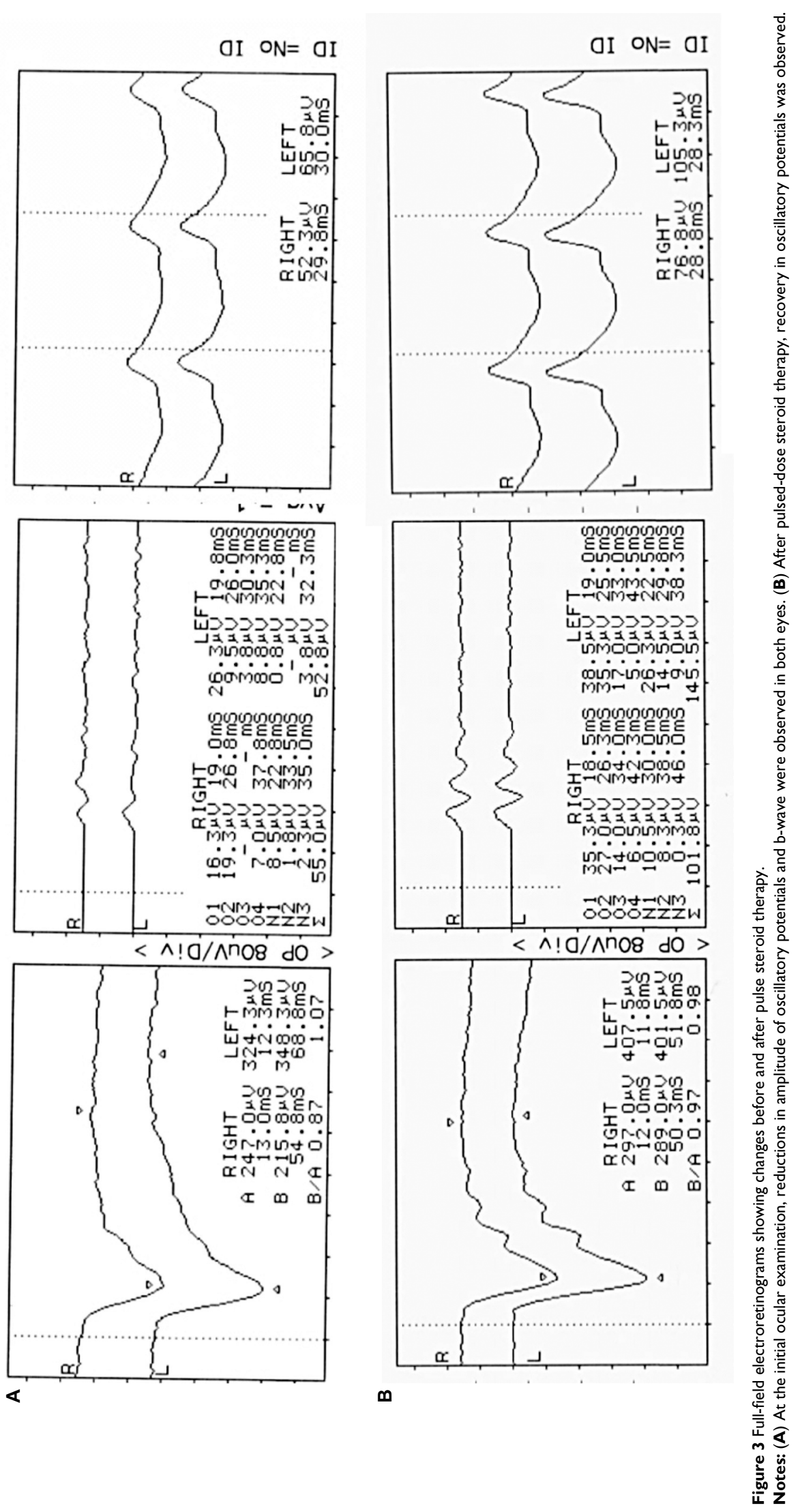


neuritis in the left eye, the patient was prescribed topical prednisolone acetate ophthalmic suspension every 5 hours and high-dose intravenous methylprednisolone $1,000 \mathrm{mg}$ daily for 3 days. One week later, anterior chamber inflammation, disk edema, and hemorrhage in the left eye were resolved. Two months after onset, his BCVA improved to 20/50 with regression of visual field defects in his left eye (Figure 2B). On a full-field ERG, recovery in oscillatory potentials was observed (Figure 3B).

\section{Discussion}

Our patient responded well to pulse steroid therapy for 3 days, with smooth recovery from blurred vision and ocular inflammation. Recovery in oscillatory potentials was also observed on a full-field ERG (Figure 3B). These findings indicate that pulse steroid therapy improved the inflammation of optic neuritis in the left eye and the activity of the secondary neurons such as horizontal cells and bipolar cells in this case. At 2-month follow-up, BCVA improved and the central scotomas in the visual field were resolved in his left eye (Figure 2B).

Lai et $\mathrm{al}^{7}$ reported the first case of acute anterior uveitis and optic neuritis after influenza $A$ infection in a 11-year-old boy. This patient was also prescribed high-dose intravenous pulse steroid therapy for 3 days and achieved good response with symptom relief and BCVA improvement.

In the present case, we performed multiplex polymerase chain reaction to detect influenza virus in the aqueous humor as the possible etiology of uveitis. However, no viruses were detected. In their case report, Lai et $\mathrm{al}^{7}$ suggested that the pathogenesis of optic neuritis and acute anterior uveitis complicating influenza A infection was not viral infection but post-viral autoimmune response, but they provided no scientific evidence. Our results obtained from the analysis of aqueous humor sample support their hypothesis because no viruses including influenza A virus were detected.

\section{Conclusion}

We described a rare case of unilateral optic neuritis and bilateral acute anterior uveitis associated with influenza A infection, which calls attention to ophthalmologists regarding the unusual association between systemic infection and ocular inflammation. Based on this case, we propose pulse steroid therapy for the treatment of anterior uveitis and optic neuritis after influenza $\mathrm{A}$ infection.

\section{Author contributions}

All authors contributed to conception and design, acquisition of data, analysis and interpretation of data. All authors drafted the article and revised it critically for important intellectual content. All authors gave final approval of the version to be published and agreed to be accountable for all aspects of the work.

\section{Disclosure}

The authors report no conflicts of interest in this work.

\section{References}

1. Jain R, Goldman RD. Novel influenza A(H1N1): clinical presentation, diagnosis, and management. Pediatr Emerg Care. 2009;25(11): 791-796.

2. Jo T, Mizota A, Hatano N, Tanaka M. Frosted branch angiitis-like fundus following presumed influenza virus type A infection. Jpn J Ophthalmol. 2006;50(6):563-564.

3. Migita M, Matsumoto T, Fujino O, Takaishi Y, Yuki N, Fukunaga Y. Two cases of influenza with impaired ocular movement. Eur J Paediatr Neurol. 2001;5(2):83-85.

4. Giraldi C, Paterni F, Cecchini S, Rama S, Mazzoni M. [Paralysis of the parasympathetic ocular nerve after influenza syndrome]. Riv Neurol. 1991;61(5):180-182.

5. Mathur SP. Macular lesion after influenza. Br J Ophthalmol. 1958; 42(11):702.

6. Gonzalez-Pola AM, Sanchez Salorio M. [Keratitis due to influenza virus]. Archivos Soc Oftalmol Hisp Am. 1951;11(9):1088-1101.

7. Lai CC, Chang YS, Li ML, Chang CM, Huang FC, Tseng SH. Acute anterior uveitis and optic neuritis as ocular complications of influenza A infection in an 11-year-old boy. J Pediatr Ophthalmol Strabismus. 2011;48: e30-e33.
The International Medical Case Reports Journal is an international, peer-reviewed open-access journal publishing original case reports from all medical specialties. Previously unpublished medical posters are also accepted relating to any area of clinical or preclinical science. Submissions should not normally exceed 2,000 words or
4 published pages including figures, diagrams and references. The manuscript management system is completely online and includes a very quick and fair peer-review system, which is all easy to use. Visit http://www.dovepress.com/testimonials.php to read real quotes from published authors. 